\title{
Cell Surface Expression of Polysialic Acid on NCAM Is a Prerequisite for Activity-Dependent Morphological Neuronal and Glial Plasticity
}

\author{
Dionysia T. Theodosis, ${ }^{1}$ Renée Bonhomme, ${ }^{1}$ Sergio Vitiello, ${ }^{1}$ Geneviève Rougon, ${ }^{2}$ and Dominique A. Poulain ${ }^{1}$ \\ 1/nstitut National de la Santé et de la Recherche Médicale U378, Institut François Magendie Université Victor Segalen \\ Bordeaux II, F33077 Bordeaux, France, and 'Centre National de la Recherche Scientifique, Unité Mixte de Recherche \\ 6545, Parc Scientifique de Luminy, F13288 Marseille, France
}

Polysialic acid (PSA) on the extracellular domain of the neural cell adhesion molecule (NCAM) reduces cell adhesion and is considered an important regulator of cell surface interactions. The hypothalamo-neurohypophysial system (HNS), whose glia, neurons, and synapses undergo striking, reversible morphological changes in response to physiological stimulation, expresses high levels of PSA-NCAM throughout life. Light and electron microscopic immunocytochemistry in normal rats and rats in which cell transport was blocked with colchicine showed that PSA-NCAM is expressed in both HNS neurons and glia, particularly at the level of astrocytic processes that envelop neuronal profiles and can undergo remodeling. Moreover, we confirmed that the overall levels of PSA-NCAM were not greatly altered by stimulation (lactation and chronic salt ingestion). Nevertheless, PSA is essential to morphological plasticity. Using comparative ultrastructural analysis, we found that, after specific enzymatic removal of PSA from NCAM by microinjection of endoneuraminidase close to the hypothalamic magnocellular nuclei in vivo, there was no apparent withdrawal of astrocytic processes nor any increase in synaptic contacts normally induced by lactation and dehydration. Our observations demonstrate, therefore, that expression of PSA on cell surfaces in the adult HNS is indispensable to its capacity for activity-dependent morphological neuronal-glial and synaptic plasticity. The carbohydrate PSA on NCAM can thus be considered a necessary permissive factor to allow neuronal and glial remodeling to occur whenever the proper inductive stimulus intervenes.

Key words: NCAM; neuronal-glial interactions; astrocytes; synaptic plasticity; hypothalamo-neurohypophysial system; lactation; osmotic stimulation
The neural cell adhesion molecule (NCAM) is represented by several isoforms that differ in their protein backbone, mode of attachment to the plasma membrane, and content of $\alpha$-2,8-linked sialic acid residues [polysialic acid (PSA)] on their extracellular domain. PSA constitutes up to $30 \%$ of the highly sialylated NCAM isoforms, designated as PSA-NCAM. Because of its negative charge and large hydrated volume, PSA on NCAM attenuates cell adhesion and has become an attractive molecular candidate to intervene in dynamic cellular changes (for review, see Rougon, 1993; Fryer and Hockfield, 1996; Rutishauser and Landmesser, 1996). For example, PSA on cell surfaces would permit cells to detach from their neighbors, thereby allowing them to undergo changes in conformation related to motility or morphological remodeling. Much evidence supports such a function in the developing CNS in which PSA-NCAM has been shown to intervene in cell migration, neurite outgrowth, and axonal fasciculation (for review, see Rutishauser and Landmesser, 1996; Yoshida et al., 1999). PSA-NCAM disappears from most of the CNS after birth, but its continued expression in neuronal systems capable of plasticity (Bonfanti et al., 1992) strongly suggests a similar role in the adult. Nevertheless, most available data are

\footnotetext{
Received Aug. 5, 1999; accepted Sept. 15, 1999.

We are grateful to Dr. C. Henderson for his critical reading of an earlier version of this manuscript. We also thank I. Svahn (Service Commun de Microscopie Université Victor Segalen Bordeaux II) for her expert photographic work.

Correspondence should be addressed to D. T. Theodosis at the above address. E-mail: dionysia.theodosis@bordeaux.inserm.fr.

Copyright (C) 1999 Society for Neuroscience $0270-6474 / 99 / 1910228-09 \$ 05.00 / 0$
}

correlative, and evidence of an actual participation is scarce. Recently, electrophysiological recordings in hippocampal slices from NCAM- or PSA-deficient mice revealed that the glycoprotein does take an active part in physiological synaptic plasticity (long-term potentiation) (Muller et al., 1996; Cremer et al., 1998). On the other hand, the abnormally small olfactory bulb in NCAM-deficient mice (Cremer et al., 1994; Ono et al., 1994) has indicated that it intervenes in neurohistogenesis and migration of PSA-NCAM-immunoreactive olfactory bulb precursors, which divide in the subventricular zone and migrate to the olfactory bulb (Bonfanti and Theodosis, 1994; Rousselot et al., 1995).

PSA-NCAM has also been implicated in synaptogenesis, but there have been no ultrastructural observations or quantitative analyses to define precisely its contribution to such structural plasticity (Rutishauser and Landmesser, 1996). A neuronal system that appears as a good model to address this question is the hypothalamo-neurohypophysial system (HNS) because it expresses high levels of PSA-NCAM throughout life (Theodosis et al., 1991; Bonfanti et al., 1992; Kiss et al., 1993; Nothias et al., 1997) and undergoes extensive morphological synaptic plasticity in response to physiological stimulation (for review, see Theodosis and Poulain, 1993; Hatton, 1997; Theodosis et al., 1998). HNS neurons, which secrete the neurohormones oxytocin or vasopressin, are grouped in well delineated areas of the hypothalamus, the supraoptic (SON) and paraventricular (PVN) nuclei; their axons project to the neurohypophysis. During lactation and chronic osmotic stimulation, there is a significant reduction in glial coverage of neuronal surfaces, and they are left directly juxtaposed 
and contacted by an increased number of synapses. The changes are reversible with cessation of stimulation (Theodosis and Poulain, 1993; Hatton, 1997; Theodosis et al., 1998).

In the present study, therefore, we first used light and electron microscopic immunocytochemistry to visualize PSA-NCAM in the rat HNS under basal and stimulated conditions of HNS secretion. To determine its cellular source more precisely, we also examined the SON of rats in which cell transport was inhibited with colchicine. We then used enzymatic removal of PSA with endoneuraminidase (endo N) (Finne and Mäkelä, 1985; Rutishauser et al., 1985) from cell surfaces in the SON of normal and stimulated rats in vivo to see whether PSA participates in the morphological changes. Because there are two bilateral SON in the hypothalamus, we were able to microinject endo $\mathrm{N}$ into one SON while keeping the other intact. This approach, coupled with comparative ultrastructural analysis, thus permitted us to monitor activity-dependent plasticity not only between groups but between two nuclei in the same animal. Finally, bilateral injections of the enzyme into two SON in lactating rats allowed us to address the question of the consequences of the morphological changes to HNS function.

\section{MATERIALS AND METHODS}

Male and female Wistar rats, at least 3 months of age and raised under controlled temperature and light conditions, were used. They were divided into the following groups according to their condition of HNS secretion: (1) normally hydrated male and virgin female rats, given food and water ad libitum; (2) salt-loaded male and female rats whose drinking water had been replaced with $2 \% \mathrm{NaCl}$ for 7-10 d; (3) females on the 19th day of gestation that, after parturition, nursed a litter of 10 pups for at least $3 \mathrm{~d}$; they were provided with food and water ad libitum; (4) lactating females that had nursed a litter of 10 pups for at least $3 \mathrm{~d}$ and were provided with food and water ad libitum; and (5) a group of normally hydrated male and female rats $(n=8)$ that, under anesthesia (a mixture of equal volumes of ketamine, chloral hydrate, and xylazine), received one injection of colchicine $(80 \mathrm{mg}$ in $2 \mu \mathrm{l})$ into a lateral ventricle; they were killed $24-72 \mathrm{hr}$ later.

\section{Immunocytochemistry}

Tissue fixation. Animals were deeply anesthetized with urethane, injected intracardially with heparin $(0.5 \mathrm{ml}, 2500 \mathrm{IU})$, and perfused with fixative composed of freshly prepared $4 \%$ paraformaldehyde and $0.1 \%$ glutaraldehyde in phosphate buffer $(0.1 \mathrm{M}, \mathrm{pH} 7.4 ; 300 \mathrm{ml}$ during $20 \mathrm{~min})$. After post-fixation in $4 \%$ paraformaldehyde overnight at $4^{\circ} \mathrm{C}$, brains were removed and cut on a vibratome to obtain frontal slices $(50-75 \mu \mathrm{m})$, which underwent immunolabeling for light and electron microscopy.

Light microscopy. For single immunostaining, standard immunofluorescence and immunoperoxidase techniques were performed on the free-floating brain sections, according to procedures described in detail in our earlier studies (Bonfanti et al., 1992; Olive et al., 1995). Briefly, after incubation in casein $(0.5 \%$ in PBS) for $1 \mathrm{hr}$ to block nonspecific sites, they were incubated in a monoclonal mouse IgM antibody that specifically recognizes PSA on NCAM. The production and characterization of the antibody are described in detail previously (Rougon et al., 1986). It is a mouse monoclonal IgM recognizing specifically $\alpha-2,8$-linked PSA with chain length greater than 12 residues. The absence of PSA in NCAM-deficient mice has provided the most compelling evidence that NCAM is indeed the major carrier of PSA in the CNS (Cremer et al., 1994; Ono et al., 1994). The antibody was used at a dilution of 1:4000$1: 6000\left(24-48 \mathrm{hr}\right.$ at $\left.4^{\circ} \mathrm{C}\right)$. Affinity-purified anti-mouse IgM immunoglobulins (Igs) conjugated to fluorescein isothiocyanate (FITC) (Immunotech, Marseille, France) (diluted 1:400) or to horseradish peroxidase (HRP) (1:50; Sigma, Les Ulis, France) were used as immunolabels. HRP reaction product was revealed either with $3,3^{\prime}$-diaminobenzidine (DAB) $(0.1 \%)$ and $0.01 \% \mathrm{H}_{2} \mathrm{O}_{2}$ or with a more sensitive method using glucose oxidase-nickel-DAB as substrate (Shu et al., 1988). The sections were examined with light microscopy (Leica, Paris, France), using bright- and dark-field optics for the HRP-containing sections and epifluorescence with appropriate filters for FITC-treated sections.

Controls included omitting the primary antibody or its substitution by diluted mouse ascites fluid containing IgM irrelevant antibodies. No specific labeling was visible on these preparations.

In some cases, double immunolabeling was performed on free-floating sections incubated for $48 \mathrm{hr}$ at $4^{\circ} \mathrm{C}$ in mixtures of primary antibodies containing anti-PSA (diluted 1:4000) and mouse monoclonal Igs raised against glial fibrillary acidic protein (GFAP) (diluted 1:500; Sigma). After careful rinsing, the sections were incubated for $2 \mathrm{hr}$ at room temperature in a mixture of fluorescent conjugates (Immunotech). Rat FITC-conjugated anti-mouse IgM Igs (diluted 1:500) were used to identify PSA immunoreactivity, whereas goat anti-mouse Igs conjugated with Texas Red (diluted 1/500) were used to visualize GFAP immunoreactivities. All preparations were examined with standard epifluorescence microscopy.

Immunoelectron microscopy. Blocks containing the SON were dissected from vibratome slices of brains obtained from different groups of rats that underwent immunoperoxidase labeling for PSA-NCAM, as described above (see also Theodosis et al., 1991). HRP reaction product was revealed with $\mathrm{DAB}$ and $\mathrm{H}_{2} \mathrm{O}_{2}$. After osmication, dehydration, block staining in uranyl acetate, and flat embedding in Epon resin, ultrathin sections were cut from selected areas and mounted on nickel grids. They were examined without any further contrast with a CM10 Philips electron microscope.

\section{In vivo endo $N$ treatment}

Unilateral injections. Stereotaxy was used to make a single microinjection of endo $\mathrm{N}$ in the vicinity of one SON (coordinates: anteroposterior,1.4 $\mathrm{mm}$; lateral, $17.5 \mathrm{~mm}$ in relation to the interaural and sagittal lines; depth, $8 \mathrm{~mm}$ from the surface of the skull). The enzyme was diluted $1: 5000$ in $2 \mu \mathrm{l}$ of artificial CSF from a stock solution containing $1 \mathrm{mg} / \mathrm{ml}$ protein. The activity of the enzyme was titrated to be $3500 \mathrm{U} / \mathrm{mg}$. The enzyme had been purified from phage K1 (Wang et al., 1994); it degrades rapidly and specifically linear polymers of sialic acid with $\alpha$-2,8-linkage with a minimum length of seven to nine residues (Rutishauser et al., 1985), characteristic of sialic acid residues associated with NCAM (Finne and Mäkelä, 1985). The injections were performed in groups of normally hydrated virgin $(n=3)$, salt-loaded $(n=5)$, and gestating $(n=3)$ rats that had been anesthetized with ketamine, chloral hydrate, and xylazine. Another group of salt-loaded rats received a similar injection of CSF alone $(n=4)$. The normally hydrated rats were fixed $6 \mathrm{~d}$ later, as were the salt-loaded rats that had been given $2 \%$ saline to drink for $6 \mathrm{~d}$ starting on the day of endo $\mathrm{N}$ injection. The gestating females were killed $12 \mathrm{~d}$ later, after they had undergone a successful parturition and had nursed a litter of 10 pups for $6-10 \mathrm{~d}$. All experimental procedures were approved by our institution's Animal Care and Use Committee.

After fixation as described above, the brains were cut on a vibratome to obtain frontal slices $(50-75 \mu \mathrm{m})$ that underwent immunofluorescence or immunoperoxidase labeling for PSA-NCAM to control the efficacy of endo $\mathrm{N}$ removal of PSA. Blocks containing the SON were dissected under microscopic control from slices of animals in which the enzyme had diffused properly (see Fig. 4). They were processed further for electron microscopy, which included post-fixation in $1 \%$ OsO4 in phosphate buffer, dehydration with increasing concentrations of ethanol, and embedding in Epon resin. To enhance contrast, they were block-stained with $1.5 \%$ uranyl acetate in $50 \%$ ethanol during dehydration. After identification of the SON in semithin sections, ultrathin sections were cut, mounted on nickel grids, contrasted with lead citrate, and examined with a Philips CM10 electron microscope. The SON on the side contralateral to the endo $\mathrm{N}$ injections was dissected and processed concomitantly.

Bilateral injections. In one group of gestating rats $(n=4)$, we used stereotaxy to make two bilateral injections of endo $\mathrm{N}$ (diluted 1:5000) in the vicinity of each SON. A group of control gestating rats $(n=4)$ that underwent no treatment were examined concurrently. The weight and water intake of the pregnant dams was recorded daily. After they gave birth, they were allowed to suckle litters of 10 pups whose weight was recorded daily. Pups and mothers were killed $8 \mathrm{~d}$ after parturition. The mothers were fixed as described above, and their brains were removed and cut on a vibratome to obtain sections that underwent immunolabeling for PSA to control the efficacy of endo $\mathrm{N}$ removal of PSA from NCAM.

\section{Quantitative ultrastructural analysis}

A comparative ultrastructural analysis was performed on tissue obtained from rats under different conditions of HNS secretion, which had received an injection of endo $\mathrm{N}$ or vehicle into the area of one SON. The analysis was performed only on tissue from animals in which the cannula 

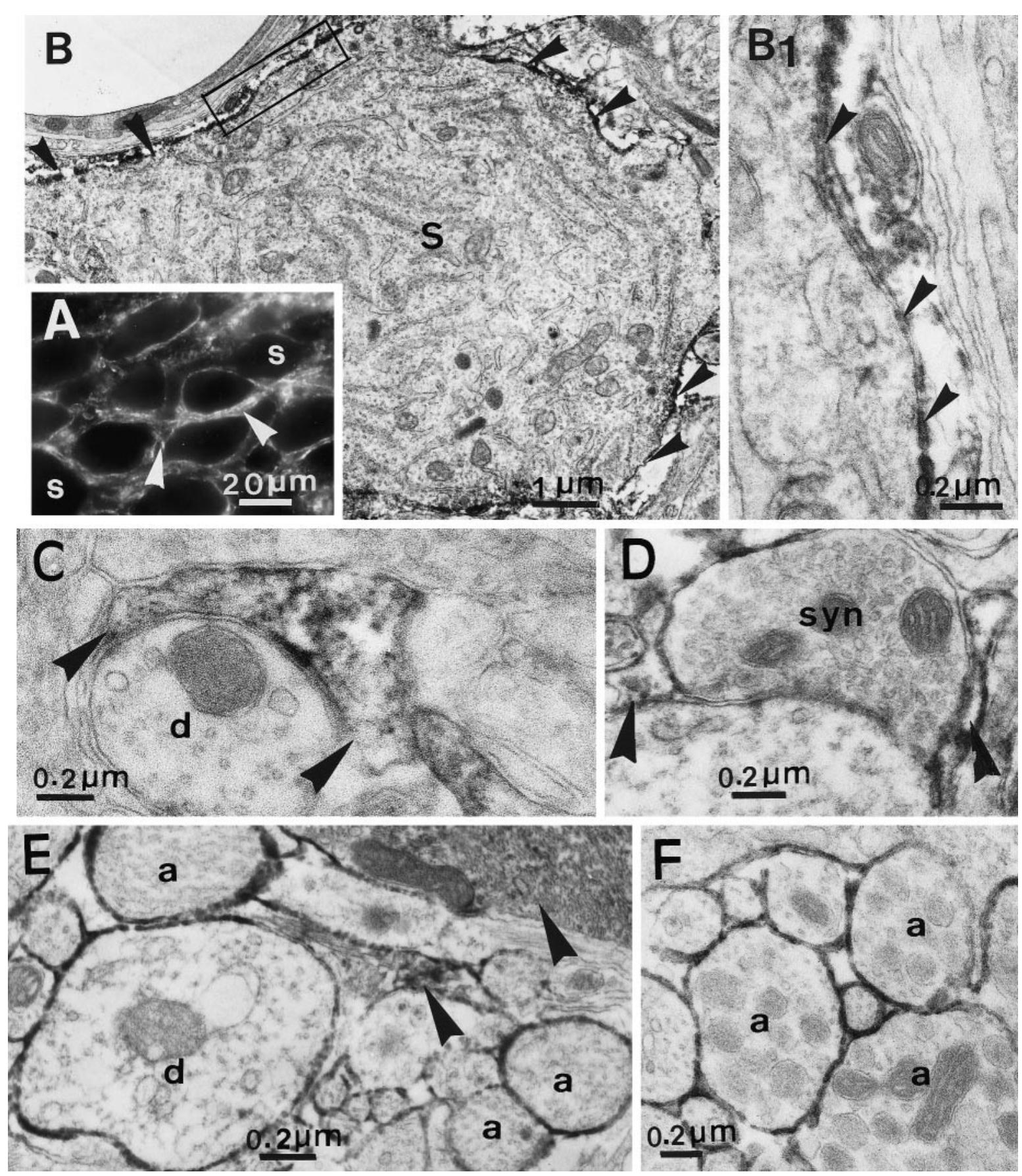

Figure 1. PSA immunoreactivity in the adult HNS under basal conditions of neurosecretion. Light microscopy of the SON $(A)$ shows an intense, discontinuous immunoreaction in the neuropile (arrowheads), around immunonegative neuronal somata $(S)$. Electron microscopy $(B-E)$ reveals that this is essentially attributable to the presence of reaction product in astrocytic processes (arrowheads) that surround immunonegative somata ( $B$ and at higher magnification in $B_{1}$ ), dendrites (d) $(C)$, and synapses $(D)$. In addition, PSA immunoreaction is visible on the surface of dendritic and axonal $(a)$ profiles, especially in the ventral region of the nucleus $(E)$. In the neurohypophysis, the surfaces of all neurosecretory axons $(a)$ are immunopositive $(F)$. $A$, Immunofluorescence; $B-F$, immunoperoxidase labeling.

had not entered the SON and in which the enzyme had diffused properly and removed all PSA immunoreactivity from inside and around the nucleus (see Fig. 4). The contralateral SON was analyzed concurrently. The analysis was performed on electron micrographs of the ventral [including the ventral glia lamina (VGL)], middle, and dorsal portions of the SON. The micrographs had a final magnification of $13,000 \times$, a magnification high enough to ensure unambiguous visualization of synapses and cell surfaces and low enough to ensure a large section area in each photograph. The profiles photographed were selected at random with reference to their position in the grid space, a type of sampling that prevented the same area from being photographed twice (see also Theodosis and Poulain, 1984). For each group, each composed of a minimum of three animals, at least nine photographs of each SON were examined, and the following parameters were obtained: (1) total number of neuronal soma and dendritic profiles; (2) number of soma and/or dendritic profiles in juxtaposition (without glial interposition) to other somata and/or dendrites; and (3) number of soma and/or dendrites contacted by the same axonal terminal simultaneously ("double" synapse). The investigator was unaware of the experimental conditions during both the photography and quantitative analysis. Raw data were 



Figure 2. PSA immunoreactivity in the HNS under stimulated conditions of neurosecretion. As shown in this example from a lactating rat, the light microscopic distribution $(A)$ in the SON is basically similar to that in unstimulated animals (compare with Fig. $1 A$ ). There is, nonetheless, some diminution of labeling associated with clusters of magnocellular somata (arrows). As seen with electron microscopy $(B, C)$, such clusters contain dendritic $\left(d_{1}, \mathrm{~d}_{2}\right)(\mathrm{B})$ and somatic $\left(S_{1}, \mathrm{~S}_{2}\right)(\mathrm{C})$ profiles whose surfaces are directly juxtaposed, with no glial interposition (between arrows). Note that PSA immunoreactivity is not associated with juxtaposed neuronal membranes, although it continues to be visible in adjacent astrocytic profiles (astro., arrowheads). $A$, Immunofluorescence; $B, C$, immunoperoxidase reaction.

analyzed using $\chi^{2}$ analysis. Statistical differences were considered significant if $p \leq 0.05$.

\section{RESULTS}

\section{PSA-NCAM is expressed by both HNS neurons and glia}

Using an antibody that specifically recognizes PSA on NCAM (Rougon et al., 1986), we confirmed that high levels of PSANCAM immunoreactivity are visible in all parts of the adult rat HNS (see also Theodosis et al., 1991; Bonfanti et al., 1992; Kiss et al., 1993). With light microscopy, the immunoreaction in the SON and PVN was characterized by an intense, discontinuous labeling around immunonegative neuronal somata (Fig. 1A). Electron microscopy showed that such a perineuronal distribution was essentially attributable to accumulation of PSA immunoreactivity on the surface and in the cytoplasm of astrocytic processes surrounding neuronal elements whose cytoplasm was free of reaction (Fig. $1 B-E$ ). PSA immunoreactivity was detected on the surface of some rare axonal and dendritic profiles, especially in the VGL (Fig. 1E). In the neurohypophysis, immunoreaction covered the surface of all neurosecretory axons and pituicytes, the astrocytic-like glia of the gland (Fig. $1 F$ ).

In accord with our earlier studies (Theodosis et al., 1991; Bonfanti et al., 1992), we found that PSA immunoreactivity did not vary greatly in its intensity or distribution in the different parts of the HNS in relation to varying conditions of neurosecretion (Fig. 2).This relative stability of PSA-NCAM expression 

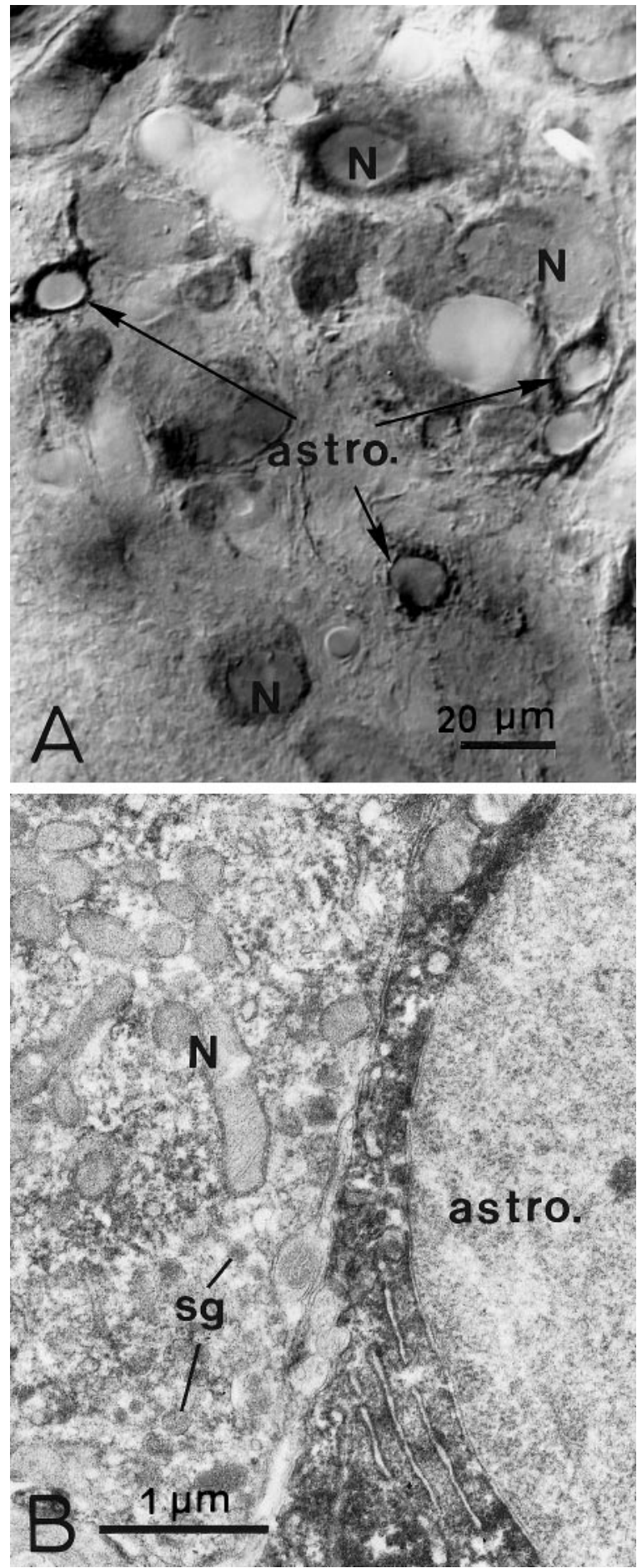

Figure 3. PSA immunoreactivity in the SON after cell transport block. Light $(A)$ and electron $(B)$ microscopy shows that, $48 \mathrm{hr}$ after one intraventricular injection of colchicine, there is less PSA immunoreaction in the neuropile. It is now heavily distributed in the cytoplasm of astrocytic cell bodies and processes (astro.) and to a variable degree in that of neuronal somata $(N)$. Note that neurosecretory granules $(s g)$ in the magnocellular soma $(N)$ are unlabeled $(B)$. Immunoperoxidase labeling.

differs considerably from the activity-dependent changes undergone by other molecules colocalized in secretory vesicles in HNS neurons. For example, during lactation and osmotic stimulation, oxytocin and vasopressin accumulate in the hypothalamic somata and decrease in neurohypophysial axons. Levels of colocalized molecules, such as chromogranins (El Majdoubi et al., 1996) or another cell adhesion molecule of the Ig superfamily, the F3 glycoprotein (Pierre et al., 1998), also increase in the hypothalamus and decrease in the neurohypophysis during strong stimulation. In the present study, we found that the only consistent modification in PSA-NCAM expression in relation to HNS activity was a reduction in labeling within clusters of neuronal somata in the nuclei of the dehydrated and lactating rats (Fig. 2A). As clearly shown with electron microscopy, these clusters consist of neuronal profiles whose surfaces are free of glial processes (Fig. $2 B, C$; see Fig. $5 A$ ), an ultrastructural feature that characterizes the stimulated SON and PVN (Theodosis and Poulain, 1993). In these clusters, we never detected any PSA immunoreactivity at the level of directly juxtaposed neuronal membranes (Fig. 2B,C).

To determine the cellular source of PSA-NCAM with more certainty, we examined tissue from animals that had been treated with colchicine, a drug often used to block axonal transport. Its action on microtubules, however, is far more extensive, and it induces a rapid blockade of newly formed material from the rough endoplasmic reticulum to the Golgi and from the Golgi to the cell surface (Malaisse and Orci, 1979; Alonso, 1988). We recently used this approach to visualize, in all HNS glia, immunoreactivity for tenascin $\mathrm{C}$, an extracellular matrix molecule that is rapidly secreted by astrocytes and usually detected only in extracellular spaces and on cell surfaces (Theodosis et al., 1997). In the present study, the distribution of PSA-NCAM in the magnocellular nuclei was visibly altered as early as $24 \mathrm{hr}$ after a single microinjection into a lateral ventricle. As seen with light and electron microscopy (Fig. 3), PSA immunolabel thus diminished in the neuropile and accumulated heavily in the cytoplasm of astrocytic cell bodies and processes. In addition, reaction product was now visible, albeit to a lesser and more variable extent, in the cytoplasm of neuronal somata. It is noteworthy that the levels of PSA immunoreactivity appeared unaltered in the neurohypophysis, even $72 \mathrm{hr}$ after intracerebral colchicine administration.

\section{In vivo endo $\mathbf{N}$ treatment of the SON}

To examine whether the expression of PSA-NCAM by HNS astrocytes and neurons is of consequence to their capacity to undergo morphological remodeling, we performed a comparative ultrastructural analysis of the SON in rats under normal (virgin) and stimulated conditions of HNS secretion known to induce plasticity (lactation and chronic salt ingestion) (Theodosis and Poulain, 1993; Hatton, 1997). Concurrent to the onset of stimulation, the animals received a single intracerebral injection of endo $\mathrm{N}$ in the vicinity of one SON; the contralateral, untreated SON served as control. After recovery from anesthesia, all animals appeared normal. For example, gestating females that had received an injection of endo $\mathrm{N}$ or vehicle gave birth as expected and then lactated in an apparently normal manner. The animals were killed, and their tissues were prepared for light and electron microscopy 6 (virgin and salt loaded groups) or 12 (lactating) d after enzyme injection.

As judged by light microscopy, endo $\mathrm{N}$ diff used in a relatively large volume of tissue around and within the $\operatorname{SON}\left(\sim 2 \mathrm{~mm}^{3}\right)$ and removed the punctate, neuropile reaction characteristic of PSA immunolabeling in this part of the brain (Fig. 4). The contralateral, uninjected side displayed the usual immunolabeling (Fig. 4). The absence of PSA immunoreactivity on cell surfaces on the side exposed to endo $\mathrm{N}$ was confirmed with electron microscopy. 


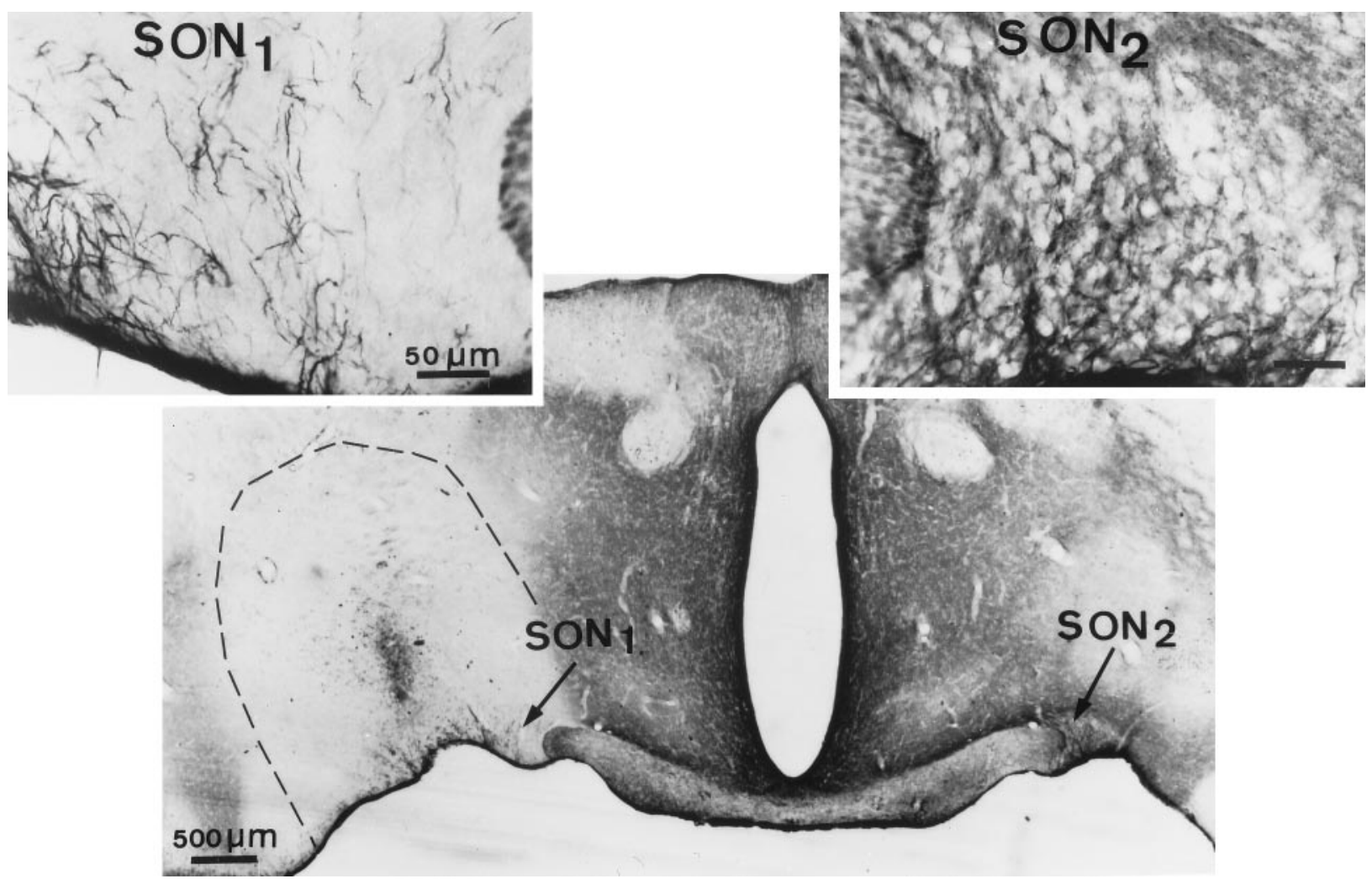

Figure 4. Effect of endo N treatment on PSA expression in the adult SON in vivo. Twelve days after one single microinjection of the enzyme in the vicinity of one SON, in this example from a lactating rat, PSA immunoreactivity disappeared from the neuropile in a large area (dotted lines) around and within the nucleus $\left(S O N_{1}\right)$. Degenerated cells in this area illustrate the point of the cannula used to deliver the enzyme. Note that some astrocytic fibers within and outside the nucleus display reaction, indicating ongoing PSA-NCAM synthesis. On the uninjected side $\left(\mathrm{SON}_{2}\right.$, and at higher magnification in the inset on the right), PSA immunoreaction appears normal in the SON and fills the neuropile around immunonegative magnocellular somata. Immunoperoxidase reaction, bright-field optics.

The neuronal-glial and synaptic changes expected to occur in response to HNS stimulation were not apparent in any part of the endo N-treated SON (Figs. 5, 6). Comparative quantitative analysis of sections that were obtained from the whole anteroposterior and dorsoventral aspects of the SON showed that the proportion of directly juxtaposed and synaptically coupled neuronal profiles in nuclei exposed to endo $\mathrm{N}$ remained low, as in the unstimulated, virgin group (Fig. 6). In contrast, neurons and glia in the SON opposite to that treated with endo $\mathrm{N}$ in the stimulated animals displayed morphological changes, to an extent similar to that reported in earlier analyses (Tweedle and Hatton, 1976; Theodosis et al., 1981; Theodosis and Poulain, 1984); close to $40 \%$ of all soma or dendritic profiles were directly juxtaposed to another neuronal profile, and $\sim 6 \%$ were coupled by the same axonal terminal (Fig. 6). It is noteworthy that injection of endo $\mathrm{N}$ into the SON of control virgin rats did not induce any morphological changes; the incidence of directly juxtaposed and synaptically coupled profiles in the SON of the endo $\mathrm{N}$-injected side were as low as that in the contralateral, uninjected side and similar to those reported previously (Tweedle and Hatton, 1976; Theodosis et al., 1981; Theodosis and Poulain, 1984).

Light and electron microscopy showed that endo $\mathrm{N}$ removed PSA from cell surfaces but did not visibly disturb cell metabolism. Moreover, it did not appear to impair PSA-NCAM synthesis because PSA immunoreactivity was detected in the cytoplasm of some astrocytic processes (Fig. 4). As expected (Eng, 1988), the mere introduction of the cannula to inject endo $\mathrm{N}$ or vehicle induced a gliosis throughout the side of the brain ipsilateral to the injection. This gliosis was similar to that described after various kinds of injury to the adult brain and was associated with the appearance of numerous activated astrocytes that displayed PSA immunoreactivity (Fig. 4). Ultrastructurally, such glia in the SON were characterized by large, thick processes filled with intermediate filaments (Fig. $5 B$ ). It was not the presence of these astrocytes that inhibited morphological remodeling in the magnocellular nuclei. In the group of salt-loaded rats exposed to vehicle alone, the SON and surrounding areas showed the same signs of reactive gliosis and damage as that seen in the endo $\mathrm{N}$ group yet continued to display the PSA immunoreaction in the neuropile and did undergo the expected morphological changes (Fig. 6).

To see whether removal of PSA from neuronal and glial surfaces in the SON is of any consequence to HNS function, we performed bilateral injections of endo $\mathrm{N}$ in the region of each of the two SON in a group of gestating female rats. PSA immunocytochemistry was used to control that the enzyme had diff used properly throughout the nuclei and that it had succeeded to remove PSA from cell surfaces. Parturition in these rats $(n=4)$ appeared to take place normally. Lactation also proceeded as expected, as judged by the mean daily weight gain of the litters. Thus, after $4 \mathrm{~d}$ of lactation (and $11 \mathrm{~d}$ after endo $\mathrm{N}$ injection), the litters of the endo $\mathrm{N}$-treated females showed a mean weight gain $(17.3 \pm 4.1 \mathrm{gm})$ that was not significantly different from that of litters $(19.2 \pm 2.0)$ that had been nursed by normal, uninjected dams $(n=4)$. 




Figure 5. Effect of endo $\mathrm{N}$ in the adult $\mathrm{SON}$ in vivo. In the $\mathrm{SON}$ of stimulated rats not exposed to endo $\mathrm{N}(A)$, there are many neuronal somata whose surfaces are directly juxtaposed, without glia interposition (between arrows); they are also coupled by the same synapse (asterisk). On the other hand, in the same animals, in the contralateral SON that had been exposed to endo $\mathrm{N}(B)$, astrocytic processes containing many glial filaments (open arrows) separate the neuronal profiles.

\section{DISCUSSION}

The present study confirms our earlier observations describing high levels of PSA-NCAM in the adult HNS and shows that PSA-NCAM is particularly conspicuous in HNS astrocytes. Neuronal expression of PSA-NCAM is well documented (Rougon, 1993), but less attention has been given to its presence in glia. Activated astrocytes that appear in response to different kinds of lesions express PSA-NCAM (Daniloff et al., 1986; Le Gal La Salle et al., 1992; Bonfanti et al., 1996), but there have been comparatively few reports of such expression in normal adult glia (Bonfanti et al., 1992; Fox et al., 1995). As shown here, strong PSA immunoreactivity was associated with HNS astrocytes, and in particular, their processes, even under normal conditions. Cell transport block with colchicine rendered such a localization more telling because it resulted in the accumulation of the glycoprotein throughout the cytoplasm of their processes and cell bodies.

HNS neurons also make PSA-NCAM, but PSA immunoreactivity was usually visible only on their axonal surfaces in the neurohypophysis and on the surface of some rare axonal and dendritic surfaces in the magnocellular nuclei. Colchicine treatment was necessary to detect PSA immunolabel in the cytoplasm of their somata, a reaction that was lighter and more variable than that seen in astrocytes. This indicates that PSA-NCAM expression is polarized in HNS neurons and that the glycoprotein is

\section{PSA-NCAM and Plasticity}
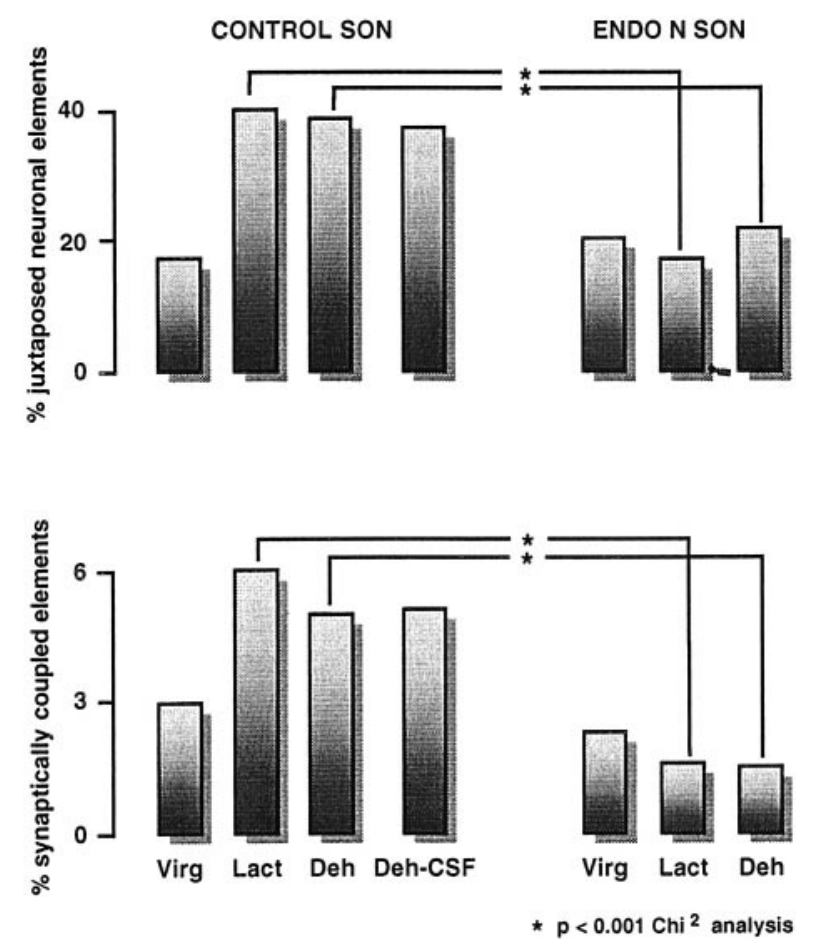

Figure 6. Effect of endo $\mathrm{N}$ on activity-dependent neuronal-glial and synaptic plasticity in the adult SON. Comparative ultrastructural analysis showed that lactation (Lact) and salt loading (Deh) induced a significant increase in the proportion of directly juxtaposed and synaptically coupled neuronal profiles (see Fig. $5 A$ ) in the SON not exposed to endo N. On the other hand, in the contralateral SON of the same animals in which endo $\mathrm{N}$ had been effective in removing surface PSA, the proportion of juxtaposed and synaptically coupled profiles is low and similar to those in unstimulated virgin rats that had or had not received endo $\mathrm{N}$ treatment. Note that, in the SON of salt-loaded rats that had received one microinjection of vehicle (Deh-CSF), plasticity occurred as expected, and the proportion of directly juxtaposed and synaptically coupled neuronal profiles is high and similar to that recorded in the SON of stimulated rats not exposed to the enzyme. $\chi^{2}$ analysis on raw data.

quickly transported to cell surfaces after synthesis, a contention supported by earlier in vitro studies (Alcaraz and Goridis, 1991). Under normal conditions, most of the glycoprotein probably occurs on cell surfaces because in vivo endo $\mathrm{N}$ treatment dramatically reduced the intense neuropile immunoreaction associated with PSA immunolabeling of the magnocellular nuclei.

That PSA-NCAM is associated with the surface of astrocytic and neuronal processes, which are considered major actors of morphological plasticity (Theodosis and Poulain, 1993; Hatton, 1997), offers strong correlative evidence that this cell adhesion molecule is important to their capacity for remodeling. Our experiments using specific enzymatic removal of PSA from NCAM in the SON provide direct evidence for this. Enzymatic manipulation of PSA-NCAM expression, especially in vivo, is considered today a most direct test for presumed PSA functions. In contrast to genetic manipulation of NCAM (Tomasiewicz et al., 1993; Cremer et al., 1994), which can reflect dysfunction because of the polypeptide itself or lead to adaptive reactions, endo $\mathrm{N}$ specifically cleaves the $\alpha$-2,8-linked sialic acid residues (Rutishauser et al., 1985) without altering the NCAM backbone (Ono et al., 1994). Such a procedure has been applied successfully 
in vivo in several neuronal systems to demonstrate intervention of PSA-NCAM in dynamic cellular processes as different as neurite outgrowth (Landmesser et al., 1990), cell migration (Ono et al., 1994; Yoshida et al., 1999), and control of circadian rhythm (Shen et al., 1997).

In the present study, such in vivo enzymatic manipulation of PSA-NCAM showed, for the first time, that PSA on NCAM is necessary for activity-dependent morphological synaptic plasticity and neuronal-glial remodeling. The existence of two bilateral SON in the hypothalamus offered the unique opportunity to have a built-in control for this kind of experimentation. As seen with light and electron microscopy, injection of endo $\mathrm{N}$ succeeded to remove PSA from a relatively large area within and around one SON, whereas the corresponding nucleus on the uninjected side continued to display the expected reaction. Moreover, as also noted by others (Landmesser et al., 1990; Shen et al., 1997; Yoshida et al., 1999), the action of the enzyme was long-lasting, being effective over 2 weeks after injection. Coupled with comparative ultrastructural analysis, this approach allowed us to show unambiguously that PSA-NCAM is essential to morphological neuronal and glial remodeling. In the endo N-treated SON, therefore, astrocytic processes continued to separate neuronal profiles, and there was no increase in synaptic inputs, despite sustained, strong stimuli that normally induce glial retraction and synapse proliferation. In contrast, these modifications occurred in the expected proportions in the contralateral nuclei not exposed to endo $\mathrm{N}$.

An obvious question is whether the ultrastructural changes are of functional consequence to the HNS. It would be surprising if the synapse proliferation associated with HNS activation is of no consequence to the activity of HNS neurons (discussed further in Theodosis and Poulain, 1993, 1996). As for astrocytic remodeling, it might serve to either allow the synaptic changes or it may have other, more direct consequences, yet to be determined (Theodosis and MacVicar, 1996). In an attempt to address this question, we here performed bilateral injections of endo $\mathrm{N}$ into the two SON in gestating animals. As expected from our unilateral endo $\mathrm{N}$ injection experiments, PSA immunoreactivity was no longer visible in the neuropile of the two nuclei, yet lactation and water intake proceeded in an apparently normal manner. This could be taken as evidence that the morphological changes are of no consequence to HNS function. It must be kept in mind, however, that the HNS is a very robust secretory system. Because of its anatomical disposition (there are four magnocellular nuclei and approximately one-third of all magnocellular neurons are interspersed anywhere in the hypothalamus), it has not been possible to totally destroy the system without killing the animals (for review, see Wakerley et al., 1994). However, extensive lesions of two nuclei leave HNS function grossly undisturbed (Wakerley et al., 1994), as we noted here after bilateral injection of endo $\mathrm{N}$ into two SON. This indicates that strong compensatory mechanisms must occur rapidly, and they could mask any subtle alterations at the electrophysiological level, for instance. Further experiments on hypothalamic slices, for example, are necessary to pursue this question in more detail.

What also remains to be elucidated is the cell mechanism by which PSA intervenes to permit morphological neuronal-glial and synaptic changes. Our observations are in agreement with a mechanism whereby large quantities of PSA on the extracellular domain of NCAM would attenuate adhesion via physical impedance or charge repulsion, thus allowing dynamic structural modifications (Rutishauser and Landmesser, 1996). Cells could then detach from their neighbors or from the extracellular matrix and be able to undergo changes in their conformation.

Whatever the molecular mechanism, PSA-NCAM cannot be considered an inductive factor for this kind of plasticity because it is continuously expressed, whatever the physiological condition, in cells that can undergo such changes. On the other hand, our observations clearly show that it is a necessary determinant for this plasticity. PSA-NCAM can be considered, therefore, as a permissive factor to allow cells to undergo remodeling whenever the proper stimulus intervenes. In the hypothalamic magnocellular nuclei, one such stimulus is oxytocin itself because its intracerebroventricular application in normal animals induced morphological changes similar to those observed under physiological stimulation (Theodosis et al., 1986). In other neuronal systems capable of similar morphological remodeling (for review, see Theodosis and Poulain, 1993) and which also express PSANCAM (Bonfanti et al., 1992), other inductive factors must intervene.

\section{REFERENCES}

Alcaraz G, Goridis C (1991) Biosynthesis and processing of polysialylated NCAM by AtT-20 cells. Eur J Cell Biol 55:165-173.

Alonso G (1988) Effects of colchicine on the intraneuronal transport of secretory material prior to the axon: a morphofunctional study in hypothalamic neurosecretory neurons of the rat. Brain Res 453:191-203.

Bonfanti L, Theodosis DT (1994) Expression of polysialylated neural cell adhesion molecule by proliferating cells in the subependymal layer of the adult rat, in its rostral extension and in the olfactory bulb. Neuroscience 62:291-305.

Bonfanti, L, Olive S, Poulain DA, Theodosis DT (1992) Mapping of the distribution of polysialylated neural cell adhesion molecule throughout the central nervous system of the adult rat: an immunohistochemical study. Neuroscience 49:419-436.

Bonfanti L, Merighi A, Theodosis DT (1996) Dorsal rhizotomy induces transient expression of the highly sialylated isoform of the neural cell adhesion molecule in neurons and astrocytes of the adult rat spinal cord. Neuroscience 74:619-623.

Cremer H, Lange R, Christoph A, Plomann M, Vopper G, Roes J, Brown R, Baldwin S, Kraemer P, Scheff S, Barthels D, Rajewsky K, Wille W (1994) Inactivation of the N-CAM gene in mice results in size reduction of the olfactory bulb and deficits in spatial learning. Nature $367: 455-459$.

Cremer H, Chazal G, Carleton A, Goridis C, Vincent JD, Lledo PM (1998) Long-term but not short-term plasticity at mossy fiber synapses is impaired in neural cell adhesion molecule-deficient mice. Proc Natl Acad Sci USA 95:13242-13247.

Daniloff JK, Levi G, Grumet M, Rieger F, Edelman GM (1986) Altered expression of neuronal cell adhesion molecules induced by nerve injury and repair. J Cell Biol 103:929-945.

El Majdoubi M, Metz-Boutigue M-H, Garcia-Sablonne P, Theodosis DT, Aunis D (1996) Immunocytochemical localization of chromogranin A in the hypothalamo-neurohypophysial system of the rat. J Neurocytol 25:405-416.

Eng LF (1988) Regulation of glial intermediate filaments in astrogliosis. In: The biochemical pathology of astrocytes (Norenberg MD, Hertz L, Schousboe A, eds), pp 79-90. New York: Liss.

Finne J, Mäkelä PH (1985) Cleavage of the polysialosyl units of brain glycoproteins by a bacteriophage endosialidase. J Biol Chem 260:1265-1270.

Fox GB, Kennedy N, Regan CM (1995) Polysialylated neural cell adhesion molecule expression by neurons and astroglial processes in the rat dentate gyrus declines dramatically with increasing age. Int J Dev Neurosci 13:663-672.

Fryer HJL, Hockfield S (1996) The role of polysialic acid and other carbohydrate polymers in neural structural plasticity. Curr Opin Neurobiol 6:113-118.

Hatton GI (1997) Function-related plasticity in the hypothalamus. Annu Rev Neurosci 20:375-397.

Kiss JZ, Wang C, Rougon G (1993) Nerve-dependent expression of high 
polysialic acid neural cell adhesion molecule in neurohypophysial astrocytes of adult rats. Neuroscience 53:213-222.

Landmesser L, Dahm L, Tang J, Rutishauser U (1990) Polysialic acid as a regulator of intramuscular nerve branching during embryonic development. Neuron 4:655-667.

Le Gal La Salle G, Rougon G, Valin A (1992) The embryonic form of neural cell surface molecule (E-NCAM) in rat hippocampus and its reexpression on glial cells following kainic acid-induced status epilepticus. J Neurosci 12:872-882.

Malaisse WJ, Orci L (1979) The role of the cytoskeleton in pancreatic B-cell function. Methods Achiev Exp Biol 9:112-136.

Muller D, Wang C, Skibo G, Toni N, Cremer H, Calaora V, Rougon G, Kiss JZ (1996) PSA-NCAM is required for activity-induced synaptic plasticity. Neuron 17:413-422.

Nothias F, Vernier P, Von Boxberg Y, Mirman S, Vincent JD (1997) Modulation of NCAM polysialylation is associated with morphofunctional modification in the hypothalamo-neurohypophysial system during lactation. Eur J Neurosci 9:1553-1565.

Olive S, Rougon G, Pierre K, Theodosis DT (1995) Expression of a glycosyl phosphatidylinositol-anchored adhesion molecule, the glycoprotein F3, in the adult rat hypothalamo-neurohypophysial system. Brain Res 689:271-280.

Ono K, Tomasiewicz H, Magnuson T, Rutishauser U (1994) N-CAM mutation inhibits tangential neuronal migration and is phenocopied by enzymatic removal of polysialic acid. Neuron 13:595-609.

Pierre K, Rougon G, Allard M, Bonhomme R, Gennarini G, Poulain DA, Theodosis DT (1998) Regulated expression of the cell adhesion glycoprotein F3 in adult hypothalamic magnocellular neurons. J Neurosci 18:5333-5343.

Rougon G (1993) Structure, metabolism and cell biology of polysialic acids. Eur J Cell Biol 61:197-207.

Rougon G, Dubois C, Buckley N, Magnani JL, Zollinger W (1986) A monoclonal antibody against meningococcus group B polysaccharides distinguishes embryonic from adult N-CAM. J Cell Biol 103:2429-2437.

Rousselot P, Lois C, Alvarez-Buylla A (1995) Embryonic (PSA) $\mathrm{N}-\mathrm{CAM}$ reveals chains of migrating neuroblasts between the lateral ventricle and the olfactory bulb of adult mice. J Comp Neurol 351:51-61

Rutishauser U, Landmesser L (1996) Polysialic acid in the vertebrate nervous system: a promoter of plasticity in cell-cell interactions. Trends Neurosci 19:422-427.

Rutishauser U, Watanabe M, Silver J, Troy FA, Vimr ER (1985) Specific alteration of NCAM-mediated cell adhesion by an endoneuraminidase. J Cell Biol 101:1842-1849.

Shen H, Watanabe M, Tomasiewicz H, Rutishauser U, Magnuson T, Glass JD (1997) Role of neural cell adhesion molecule and polysialic acid in mouse circadian clock function. J Neurosci 17:5221-5229.
Shu S, Ju G, Fan L (1988) The glucose oxidase-DAB-nickel method in peroxidase histochemistry of the nervous system. Neurosci Lett 85:169-171.

Theodosis DT, MacVicar BA (1996) Neuron-glia interactions in the hypothalamus and pituitary. Trends Neurosci 19:363-367.

Theodosis DT, Poulain DA (1984) Evidence for structural plasticity in the supraoptic nucleus of the rat hypothalamus in relation to gestation and lactation. Neuroscience 11:183-193.

Theodosis DT, Poulain DA (1993) Activity-dependent neuronal-glial and synaptic plasticity in the adult mammalian hypothalamus. Neuroscience 57:501-535.

Theodosis DT, Poulain DA (1996) Pulsatile neuronal activity and structural synaptic plasticity of the adult oxytocinergic system. Curr Opin Endocrinol Diab 3:164-170.

Theodosis DT, Poulain DA, Vincent JD (1981) Possible morphological bases for synchronisation of neuronal firing in the rat supraoptic nucleus during lactation. Neuroscience 6:919-929.

Theodosis DT, Montagnese C, Rodriguez F, Vincent JD, Poulain DA (1986) Oxytocin induces morphological plasticity in the adult hypothalamo-neurohypophysial system. Nature 322:738-740.

Theodosis DT, Rougon G, Poulain DA (1991) Retention of embryonic features by an adult neuronal system capable of plasticity: Polysialylated N-CAM in the hypothalamo-neurohypophysial system. Proc Natl Acad Sci USA 88:5494-5498.

Theodosis DT, Pierre K, Cadoret MA, Allard M, Faissner A, DA Poulain (1997) Expression of high levels of the extracellular matrix glycoprotein, tenascin- $\mathrm{C}$, in the normal adult hypothalamoneurohypophysial system. J Comp Neurol 379:386-398.

Theodosis DT, El Majdoubi M, Pierre K, Poulain DA (1998) Factors governing activity-dependent structural plasticity of the hypothalamoneurohypophysial system. Cell Mol Neurobiol 18:285-298.

Tomasiewicz H, Ono K, Yee D, Thompson C, Goridis C, Rutishauser U, Magnuson T (1993) Genetic deletion of a neural cell adhesion molecule variant (N-CAM-180) produces distinct defects in the central nervous system. Neuron 11:1163-1174.

Tweedle CD, Hatton GL (1976) Ultrastructural comparisons of neurons of supraoptic and circularis nuclei in normal and dehydrated rats. Brain Res Bull 1:103-121.

Wakerley JB, Clarke G, Summerlee AJS (1994) Milk ejection and its control. In: The physiology of reproduction, Ed 2 (Knobil E, Neill JD, eds), pp 2283-2322. New York: Raven

Wang C, Rougon G, Kiss JZ (1994) Requirement of polysialic acid for the migration of the O-2A glial progenitor cell from neurohypophysial explants. J Neurosci 14:4446-4457.

Yoshida K, Rutishauser U, Crandall JE, Schwarting GA (1999) Polysialic acid facilitates migration of luteinizing migration of luteinizing hormone-releasing hormone neurons on vomeronasal axons. J Neurosci 19:794-801. 